\title{
Proton pump inhibitor is a risk factor for recurrence of common bile duct stones after endoscopic sphincterotomy - propensity score matching analysis
}

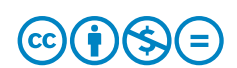

\author{
Authors \\ Nobuhiko Fukuba ${ }^{1}$, Shunji Ishihara ${ }^{1}$, Hiroki Sonoyama ${ }^{1}$, Noritsugu \\ Yamashita ${ }^{1}$, Masahito Aimi ${ }^{1}$, Yoshiyuki Mishima' ${ }^{1}$ Tsuyoshi Mishiro ${ }^{1}$, \\ Hiroshi Tobita ${ }^{1}$, Koutarou Shibagaki ${ }^{1}$, Naoki Oshima ${ }^{1}$, Ichiro \\ Moriyama ${ }^{2}$, Kousaku Kawashima ${ }^{1}$, Tatsuya Miyake ${ }^{1}$, Norihisa \\ Ishimura ${ }^{1}$, Shuichi Sato ${ }^{3}$, Yoshikazu Kinoshita ${ }^{1}$ \\ Institutions \\ 1 Department of Internal Medicine II, Shimane University School \\ of Medicine, Japan \\ 2 Division of Cancer Center, Shimane University Hospital, Japan \\ 3 Division of Gastrointestinal Endoscopy, Shimane University \\ Hospital, Japan
}

submitted 29.3.2016

accepted after revision 1.2 .2017

\begin{abstract}
Bibliography
DOI http://dx.doi.org/10.1055/s-0043-102936 |

Endoscopy International Open 2017; 05: E291-E296

(c) Georg Thieme Verlag KG Stuttgart · New York ISSN 2364-3722
\end{abstract}

Corresponding author

Nobuhiko Fukuba, MD, Department of Internal Medicine II, Shimane University Faculty of Medicine, 89-1, Enya-cho, Izumo, Shimane, Japan

fnat98@yahoo.co.jp

\begin{abstract}
Background and study aims Recurrence of common bile duct stones (CBDS) in patients treated with endoscopic sphincterotomy (ES) can lead to deterioration in their quality of life. Although the pathology and related factors are unclear, we speculated that proton pump inhibiter (PPI) administration increases the risk of CBDS recurrence by altering the bacterial mixture in the bile duct.

Patients and methods The primary endpoint of this retrospective study was recurrence-free period. Several independent variables considered to have a relationship with CBDS recurrence including PPI use were analyzed using a COX proportional hazard model, with potential risk factors then evaluated by propensity score matching analysis.

Results A total of 219 patients were analyzed, with CBDS recurrence found in 44 . Analysis of variables using a COX proportional hazard model demonstrated that use of PPIs and ursodeoxycholic acid (UDCA), as well as the presence of periampullary diverticula (PD) each had a hazard ratio (HR) value greater than 1 (HR 2.2, $P=$ 0.007; HR 2.0, $P=0.02$; HR 1.9, $P=0.07$; respectively). Furthermore, propensity score matching analysis revealed that the mean recurrence-free period in the oral PPI cohort was significantly shorter as compared with the non-PPI cohort (1613 vs. 2587 days, $P=0.014$ ). In contrast, neither UDCA administration nor PD presence was found to be a significant factor in that analysis ( 1557 vs. 1654 days, $P=0.508 ; 1169$ vs. 2011 days, $P=0.121$; respectively).

Conclusion Our results showed that oral PPI administration is a risk factor for CBDS recurrence in patients who undergo ES.
\end{abstract}

\section{Introduction}

Patients with common bile duct stones (CBDS) are frequently encountered in clinical situations, with cholangitis development occurring in some, followed by sepsis. Endoscopic sphincterotomy (ES) was developed in the $1970 \mathrm{~s}$ as a procedure for removal of stones from the CBD without the need for surgery $[1,2]$. Although it has become the standard therapy method for CBDS [3], long-term complications following that procedure have been reported. In a review of 371 patients, Saito et al. found that CBDS recurrence occurred in $9.7 \%$ during a mean follow-up period of 7.7 years [4]. Furthermore, Ando et al. reported $111(11 \%)$ cases of recurrence in 1042 consecutive patients with CBDS who underwent ES during a median period of 7.5 years [5] and a more recent study reported a recurrence rate of $8.5 \%$ over a median period of 7.5 years [6]. Thus, CBDS recurrence in patients treated with ES is not uncommon and may cause deterioration in their quality of life.

Previous reports have shown that pneumobilia, gall bladder status, use of lithotripsy, presence of periampullary diverticula $(P D)$, and presence of a dilated bile duct were risk factors for CBDS recurrence $[4,5,7-9]$. However, no known reports clarifying whether oral medicines including PPIs cause such recurrence have been presented. Although PPIs are commonly prescribed drugs for reducing gastric acid production, several studies have found that long-term recipients experience various complications, especially small intestine bacterial overgrowth (SIBO) [10-14]. We speculated that PPI administration increases the risk of CBD stone recurrence by altering the bacterial mixture in the bile duct in association with SIBO. To examine this possibility, in the current study we compared PPI use with other risk factors previously reported using a COX proportional hazard model. However, that method is not considered 
suitable for analyzing numerous independent variables or making adjustments based on subject background factors, thus we also used propensity score matching analysis to investigate candidate risk factors, as that has been shown capable of adjusting for a large number of characteristics other than the factor under evaluation in the examined subjects [15]. In addition, this method has also been used recently in a variety of clinical and statistical investigations [16]. For the current analysis, we focused on patients treated with ES in order to reveal risk factors related to CBDS recurrence using a COX proportional hazard model and propensity score matching analysis.

\section{Patients and methods}

\section{Study design}

This study was retrospective and conducted at Shimane University Hospital, Japan. The primary endpoint was CBDS recurrence-free period with or without the presence of risk factors. First, we determined factors related to CBDS recurrence using a COX proportional hazard model, with those findings evaluated by propensity score matching analysis to reduce confounding bias. The study protocol was approved by the Institutional Review Board of Shimane University.

\section{Subjects}

Consecutive patients who underwent ES from October 2005 to March 2015 that resulted in complete resolution of CBDS were initially enrolled. Those who received endoscopic papillary large balloon dilatation in addition to ES were included, whereas patients who underwent endoscopic papillary balloon dilatation alone were excluded. In addition, patients treated by major gastrointestinal surgery with an altered route of food passage and those with a bile duct stricture, such as from pancreatobiliary malignancy or postoperative scarring, were also excluded.

\section{Candidate risk factors}

Medical charts of the enrolled subjects were retrospectively reviewed, with patient characteristics, oral drug use, and underlying disease noted as candidate risk factors. Presence of PD was diagnosed according to endoscopic findings obtained when the ES procedure was performed. The drug-administrated group included patients who took an oral drug during more than two-thirds of the follow-up period after undergoing ES.

\section{Definition of CBDS recurrence}

Date of CBDS recurrence was determined as the date of the imaging test, such as abdominal ultrasonography, ultrasonic endoscopy, computed tomography, or magnetic resonance cholangiopancreatography, that showed evidence of CBDS, or the date on which cholangitis was diagnosed by serum liver or biliary function test findings. Patients who had treatment interrupted, or who died or were transferred during the study period were treated as censored cases from the final visit day.

\section{Statistical analysis}

A COX proportional hazard model was used with a forced entry method and the appropriate number of independent variables was estimated based on the number of events. To adjust for the numerous characteristics, propensity score matching was performed using independent variables with a hazard ratio $>1$ in COX proportional hazard model analysis. The propensity score was determined using a multiple logistic regression model with a forced entry method, and then rounded up to 3 decimal places using the rounding function. Next, using propensity score results, we extracted 53 matching pairs for whom PPIs were and were not actually prescribed. In addition, we also extracted pairs for UDCA and PD. Pair matching was done with a nearest neighbor search with the additional payment syntax. KaplanMeier curves for cumulative non-incidence of CBDS were produced for cohorts with and without candidate risk factors using a log-rank test. All analyses were performed according to the intention-to-treat principle, with the results calculated using SPSS (ver. 19) and $P$ values $<0.05$ considered to indicate significance.

\section{Results}

\section{Subjects}

A total of 270 patients underwent endoscopic therapy for CBDS during the study period, of whom 51 were excluded (altered gastrointestinal tract route, $n=17$; endoscopic papilla balloon dilatation, $n=14$; incomplete removal of CBDS, $n=20$ ), thus 219 were subjected to analysis. All underwent ES with a medium range incision. Patient baseline characteristics, underlying diseases, prescribed oral drugs, and use of mechanical lithotripsy are shown in - Table 1 . CBDS recurrence was noted in $20 \%$ (44/219) of all patients. Of the 73 patients administered PPIs (mean period 338 days), $32 \%$ (23/73) suffered from recurrence.

\section{COX proportional hazard model}

We selected the following 5 independent variables for our analysis: history of cholecystectomy, use of mechanical lithotripsy, presence of PD, and PPI and UDCA administrations. Subsequent analysis findings revealed that PPI and UDCA use and presence of PD each had a hazard ratio (HR) value greater than 1 (HR 2.2, $P=0.007$; HR 2.0, $P=0.02$; HR 1.9, $P=0.07$; respectively) ( $\triangleright$ Table 2).

\section{Calculation of propensity scores for PPI, UDCA, and PD}

We then adjusted for the numerous characteristics using propensity score matching and examined whether the 3 independent variables were risk factors for CBDS recurrence. Propensity scores for PPI and UDCA administration, and presence of PD were calculated on a case-by-case basis by multinomial logistic regression using 10 apparently relevant variables (age, gender, history of cholecystectomy, antiplatelet medicine use, history of cerebral infarction, coronary disease, hypertension, diabetes, hypercholesterolemia, hypertriglyceridemia). As a 
- Table 1 Baseline characteristics of patients $(n=219)$.

\begin{tabular}{|c|c|c|}
\hline Median age in years (IQR) & 77 & $(68-82)$ \\
\hline Female gender & 85 & $(39 \%)$ \\
\hline Periampullary diverticula & 44 & $(20 \%)$ \\
\hline Post-cholecystectomy & 27 & $(12 \%)$ \\
\hline \multicolumn{3}{|l|}{ Past illness } \\
\hline History of cerebral infarction & 21 & $(9.6 \%)$ \\
\hline History of coronary disease & 16 & $(7.3 \%)$ \\
\hline \multicolumn{3}{|l|}{ Present illness } \\
\hline Hypertension & 96 & $(44 \%)$ \\
\hline Hypercholesterolemia & 39 & $(18 \%)$ \\
\hline Hypertriglyceridemia & 4 & $(1.4 \%)$ \\
\hline Diabetes & 37 & $(17 \%)$ \\
\hline \multicolumn{3}{|l|}{ Drugs } \\
\hline Antiplatelet & 38 & $(17 \%)$ \\
\hline PPI & 73 & $(33 \%)$ \\
\hline UDCA & 65 & $(29 \%)$ \\
\hline Mechanical lithotripsy & 38 & $(17 \%)$ \\
\hline Median observation period in days (IQR) & 454 & $(171-1082)$ \\
\hline CBDS recurrence & 44 & $(20 \%)$ \\
\hline
\end{tabular}

result, area under the curve (AUC) values for propensity scores for PPI and UDCA administration, and presence of PD were $0.658,0.640$, and 0.630 , respectively. Although the AUC values for the propensity scores indicated low to moderate accuracy, it was considered that a value $\geq 0.6$ was within the limit of tolerance. Hosmer-Lemeshow test results of these findings did not reveal significant differences $\left(X^{2}=9.692, d f=8, P=0.287 ; X^{2}=\right.$ $3.478, d f=8, P=0.901 ;=10.567, d f=8, P=0.227$; respectively).

\section{Propensity score matching analysis for PPI and UDCA use, and presence of PD}

To extract pairs from the 2 groups after adjusting for factors other than those of interest, 1-to-1 matching between the oral PPI and non-PPI cohorts was conducted based on propensity score, which resulted in extraction of 53 pairs to compose cohorts with and without PPI administration. Kaplan-Meier curves for cumulative non-incidence of CBDS recurrence in the matching cohorts are shown in > Fig. 1. The 20 groups were matched based on propensity score matching for the factor of interest and then adjusted for the other factors. The estimated mean recurrence-free period was shorter in the oral PPI cohort (1613 vs. 2587 days), and log-rank test results showed a significant difference between the groups with and without PPI administration $(P=0.014)$.

On the other hand, administration of UDCA and presence of PD were found to be significant factors in 65 and 44 subjects, respectively. From the cohorts with and without each variable,
- Table 2 Independent variables related to CBDS recurrence shown by multivariate COX proportional hazard model.

\begin{tabular}{|l|l|l|l|l|}
\hline $\begin{array}{l}\text { Independent } \\
\text { variables }\end{array}$ & HR & \multicolumn{2}{|c|}{$\mathbf{9 5 \% \text { confidence interval }}$} & P value \\
\cline { 1 - 4 } $\begin{array}{l}\text { Cholecys- } \\
\text { tectomy }\end{array}$ & 0.708 & 0.252 & 1.990 & 0.513 \\
\hline $\begin{array}{l}\text { Mechanical } \\
\text { lithotripsy }\end{array}$ & 0.659 & 0.306 & 1.418 & 0.286 \\
\hline PD & 1.873 & 0.957 & 3.668 & 0.067 \\
\hline PPI & 2.232 & 1.241 & 4.015 & 0.007 \\
\hline UDCA & 1.959 & 1.119 & 3.428 & 0.019 \\
\hline
\end{tabular}

PD, periampullary diverticula; PPI, proton pump inhibitor; ursodeoxycholic acid

we extracted 26 and 28 pairs, respectively, and subjected them to Kaplan-Meier analysis, which found no significant difference between them in regard to recurrence-free period (1557 vs. 1654 days, $P=0.508 ; 1169$ vs. 2011 days, $P=0.121$; respectively) ( Fig. 2, $\triangleright$ Fig. 3).

\section{Discussion}

This study was undertaken to answer clinical questions regarding why CBDS recurrence occurs after ES. Although some suspected risk factors have been reported [4, 5, 7 -9], few investigations have been conducted to clarify whether oral medicine use increases the risk of recurrence. We speculated that PPI administration may increase the risk of CBDS recurrence and evaluated candidate risk factors using a COX proportional hazard model, which showed that PPI and UDCA administrations, as well as presence of PD were related to the recurrence-free period in the present subjects. Thereafter, propensity score matching analysis indicated that oral PPI administration alone was an independent factor to increase risk of CBDS recurrence. Although various factors related to biliary complications in patients who have undergone ES have been identified, few studies have considered oral drugs or underlying diseases in their evaluations of risk factors related to CBDS recurrence in those cases. The current COX proportional hazard model revealed that PPI was a risk factor, although several characteristics known to affect CBDS recurrence were not included in the analysis, due to the limitation regarding number of variables. To solve that issue, we used propensity score matching in the current study. A pitfall of propensity score matching analysis is reduction in sample size. On the other hand, it can adjust for numerous characteristics by consolidating many covariates into a propensity score and can reduce bias to a greater degree as compared with a COX proportional hazard model. This is an important advantage of this method and we considered that the benefit of reducing bias was greater as compared to the disadvantage of sample size reduction. Therefore, we performed propensity score matching analysis in addition to a COX proportional hazard model in the current study. 


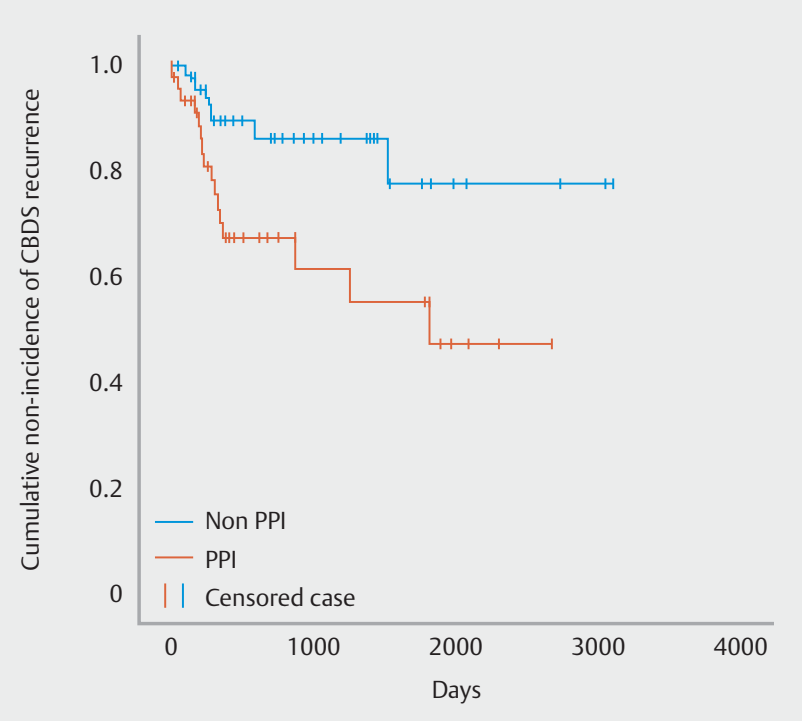

- Fig. 1 Kaplan-Meier curves showing cumulative non-incidence of CBDS recurrence in matching cohorts with and without PPI administration. The estimated mean recurrence-free period was significantly shorter in the PPI oral cohort (1613 vs. 2587 days, $P=0.014$ ).

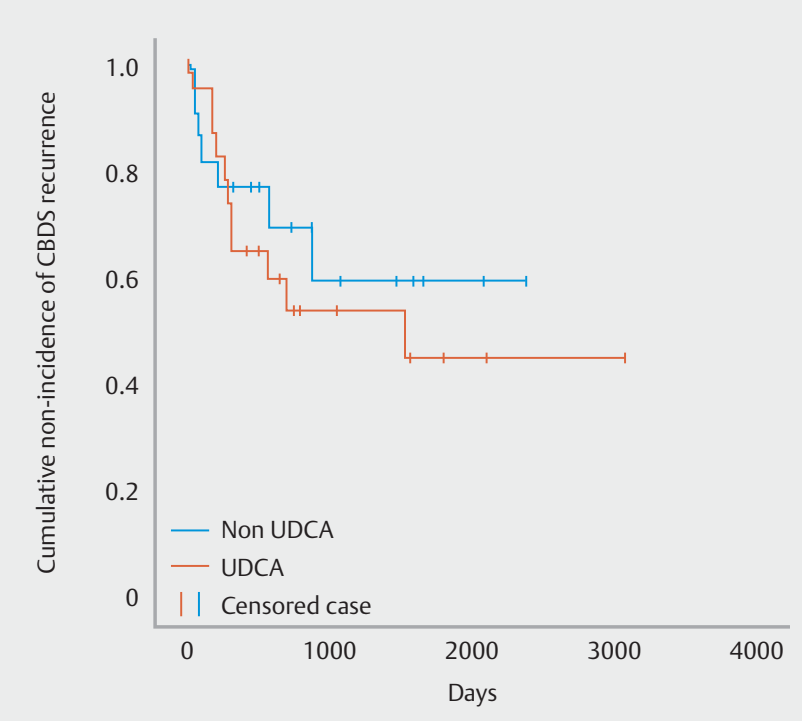

- Fig. 2 Kaplan-Meier curves showing cumulative non-incidence of CBDS recurrence in matching cohorts with and without UDCA. There was no significant difference between the cohorts (1557 vs. 1654 days, $P=0.508)$.

Findings of COX proportional hazard model analysis indicated that UDCA administration was related to a shortened recurrence-free period in the present subjects. However, UDCA is the most widely used therapeutic agent for treatment of cholestatic hepatopathies [17 - 19] and the reason for this discrepancy is unclear. Interestingly, Akiyama et al. also reported that recurrent CBDS was associated with UDCA treatment and suggested that the drug causes stone formation via an unknown mechanism [20]. Although UDCA use may be shown to be a risk factor

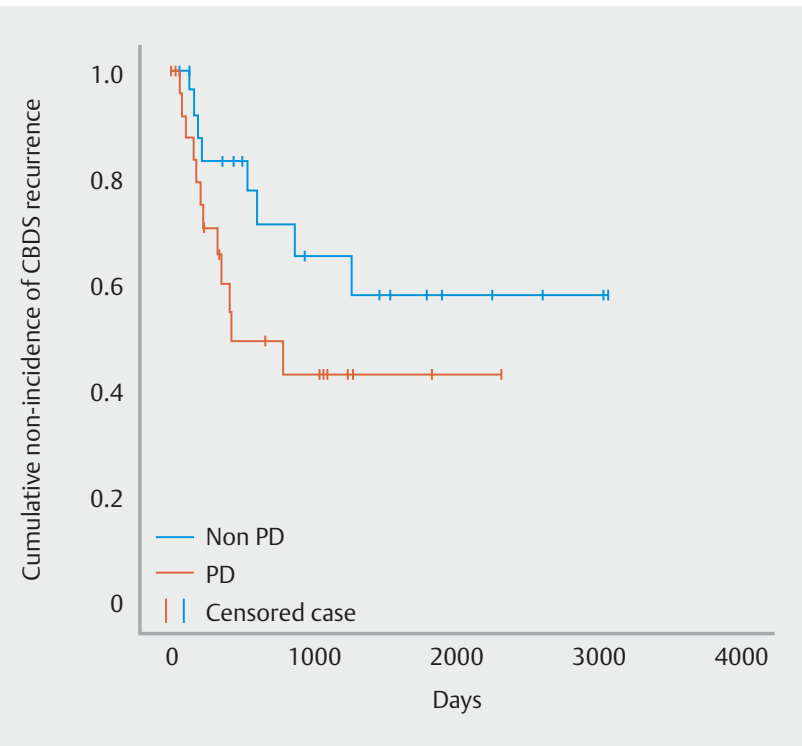

- Fig. 3 Kaplan-Meier curves showing cumulative non-incidence of CBDS recurrence in matching cohorts with and without PD. There was no significant difference between the cohorts (1169 vs. 2011 days, $P=0.121$ ).

for CBDS recurrence in a future study, the present propensity score matching analysis found no difference between the recurrence-free periods in patients with and without UDCA administration, suggesting that UDCA itself is not a causative factor.

In addition, our analysis using a COX proportional hazard model indicated that presence of PD was also related to a shortened recurrence-free period, although the difference was not significant. PD has been reported to be a risk factor for development of CBDS, while intra-diverticular overgrowth of bacteria and bile flow disturbance are also believed to increase its incidence $[21,22]$. As noted in those studies, clinical presence of PD is thought to be a risk factor and the hazard ratio was 1.873 in the current COX proportional hazard model findings, thus we cannot deny that possibility. However, the current propensity score matching analysis found no significant difference between cohorts with and without PD, although it is possible that the low sample size due to matching analysis reduced the power of the calculation.

The current COX proportional hazard model and propensity score results revealed that PPI administration increases CBDS recurrence in patients who undergo ES. Although PPIs are commonly prescribed and have been found to have a low rate of adverse effects, there are recent reports showing that long-term recipients experience various complications, such as pneumonia, Clostridium difficile infection, and collagenous colitis, as well as malabsorption of vitamin B12, Mg, and Ca[10-12,23]. Alternatively, SIBO is also considered to occur in patients receiving PPIs [13], as that was detected in $50 \%$ of affected patients as compared to $6 \%$ of healthy control subjects in a previous study [14]. Bacterial colonization correlated with formation of gallstones as well as bacterial infection have been proposed as key factors in the pathogenesis of pigment gallstones 
[24-25]. Furthermore, Rongchun et al. reported that the rate of duodenobiliary reflux (DBR) was increased in patients with recurrent $C B D S$, as the function of the sphincter of Oddi was found to be significantly worse in a post-ES group as compared to a post-EPBD group [26]. Damage to the sphincter of Oddi after an ES procedure may lead to biliary infection and stone recurrence secondary to reflux of duodenal contents into the bile duct $[27,28]$. Also, SIBO induced by PPI use might lead to bacterial infection of the bile duct via DBR in patients who have undergone ES. The present findings suggest that oral PPI administration increases the risk of CBDS recurrence in patients who undergo ES via bacterial overgrowth in the small intestine, especially the duodenum. On the other hand, there are no known published findings regarding cultured bacteria obtained from the duodenum of CBDS patients with or without PPI administration, and such a study is needed.

Our study is limited by the small sample size, which did not allow for adequate analysis of additional candidate risk factors related to CBDS recurrence. Furthermore, in this retrospective investigation, gallbladder status could not be included due to the small sample size, thus CBDS recurrence caused by escape of gallbladder stones could not be distinguished from CBDS recurrence derived from duodenobiliary reflux. However, it is difficult to determine the type of stones in patients with CBDS recurrence. Accordingly, a multicenter study with a larger sample size is needed in order to confirm our findings.

Incidence of CBDS recurrence was $20 \%$ in this study, higher than that noted in other reports [6], which may have been the result of inclusion of cholangitis among the criteria to determine recurrence. Although cholangitis should be distinguished from CBDS, it is difficult to differentiate those conditions clinically, because cholangitis is often complicated in patients with CBDS. Therefore, cholangitis was included with recurrence of CBDS as the primary endpoint of the present study.

\section{Conclusion}

In summary, our findings revealed that PPI administration is a risk factor for CBDS recurrence in patients who have been treated with ES. Although PPIs are the most commonly prescribed drugs for these cases and indispensable in clinical practice, continuation of unnecessary administration should be avoided. Notably, patients who have undergone an ES procedure should be carefully monitored.

\section{Competing interests}

None

\section{References}

[1] Classen M, Demling L. Endoscopic sphincterotomy of the papilla of vater and extraction of stones from the choledochal duct. Dtsch Med Wochenschr 1974; 99: 496-497
[2] Kawai K, Akasaka Y, Murakami K et al. Endoscopic sphincterotomy of the ampulla of Vater. Gastrointest Endosc 1974; 20: 148-151

[3] Trikudanathan G, Arain MA, Attam R et al. Advances in the endoscopic management of common bile duct stones. Nat Rev Gastroenterol Hepatol 2014; 11: 535 - 544

[4] Saito M, Tsuyuguchi T, Yamaguchi T et al. Long-term outcome of endoscopic papillotomy for choledocholithiasis with cholecystolithiasis. Gastrointest Endosc 2000; 51: 540- 545

[5] Ando T, Tsuyuguchi T, Okugawa T et al. Risk factors for recurrent bile duct stones after endoscopic papillotomy. Gut 2003; 52: 116-121

[6] Doi S, Yasuda I, Mukai T et al. Comparison of long-term outcomes after endoscopic sphincterotomy versus endoscopic papillary balloon dilation: A propensity score-based cohort analysis. J Gastroenterol 2013; 48: 1090 - 1096

[7] Lu Y, Wu J-C, Liu L et al. Short-term and long-term outcomes after endoscopic sphincterotomy versus endoscopic papillary balloon dilation for bile duct stones. Eur J Gastroenterol Hepatol 2014; 26: 1367 1373

[8] Kim KY, Han J, Kim HG et al. Late complications and stone recurrence rates after bile duct stone removal by endoscopic sphincterotomy and large balloon dilation are similar to those after endoscopic sphincterotomy alone. Clin Endosc 2013; 46: 637-642

[9] Sugiyama M, Atomi Y. Risk factors predictive of late complications after endoscopic sphincterotomy for bile duct stones: long-term (more than 10 years) follow-up study. Am J Gastroenterol 2002; 97: $2763-2767$

[10] Lam JR, Schneider JL, Zhao W et al. Proton pump inhibitor and histamine 2 receptor antagonist use and vitamin B12 deficiency. JAMA 2013; 310: 2435-2442

[11] Abraham NS. Proton pump inhibitors: potential adverse effects. Curr Opin Gastroenterol 2012; 28: 615-620

[12] Leontiadis GI, Moayyedi P. Proton pump inhibitors and risk of bone fractures. Curr Treat Options Gastroenterol 2014; 12: 414-423

[13] Lo W, Chan WW. Proton Pump Inhibitor Use and the Risk of Small Intestinal Bacterial Overgrowth: A Meta-analysis. Clin Gastroenterol Hepatol 2013; 11: $483-490$

[14] Lombardo L, Foti M, Ruggia O et al. Increased incidence of small intestinal bacterial overgrowth during proton pump inhibitor therapy. Clin Gastroenterol Hepatol 2010; 8: 504-508

[15] Rosenbaum PR, Rubin DB, Central THE et al. The central role of the propensity score in observational studies for causal effects. Biometrika 1983; 70: $41-55$

[16] Dehejia R, Wahba S. Propensity Score Matching Methods for Nonexperimental Studies. Rev Econ Stat 2002; 84: 151 - 161

[17] Calmus Y, Poupon R. Ursodeoxycholic Acid (UDCA) in the treatment of chronic cholestatic diseases. Biochimie 1991; 73: 1335-1338

[18] Guarino MPL, Cocca S, Altomare A et al. Ursodeoxycholic acid therapy in gallbladder disease, a story not yet completed. World J Gastroenterol 2013; 19: 5029-5034

[19] Roma MG, Toledo FD, Boaglio AC et al. Ursodeoxycholic acid in cholestasis: linking action mechanisms to therapeutic applications. Clin Sci (Lond) 2011; 121: 523-544

[20] Akiyama S, Imamura T, Tamura T et al. Recurrent common bile duct stones composed of ursodeoxycholic acid: A report of four cases. Intern Med 2014; 53: 2489-2492

[21] Li X, Zhu K, Zhang L et al. Periampullary diverticulum may be an important factor for the occurrence and recurrence of bile duct stones. World J Surg 2012; 36: 2666-2669

[22] Kim CW, Chang JH, Kim JH et al. Size and type of periampullary duodenal diverticula are associated with bile duct diameter and recurrence of bile duct stones. J Gastroenterol Hepatol 2013; 28: 893-898 
[23] Reilly JP. Safety profile of the proton-pump inhibitors. Am J Health Syst Pharm 1999; 56: S11-S17

[24] Peng Y, Yang Y, Liu Y et al. Cholesterol gallstones and bile host diverse bacterial communities with potential to promote the formation of gallstones. Microb Pathog 2015; 83-84: 57-63

[25] Maki T. Pathogenesis of calcium bilirubinate gallstone: role of E. coli, beta-glucuronidase and coagulation by inorganic ions, polyelectrolytes and agitation. Ann Surg 1966; 164: $90-100$
[26] Yasuda I, Tomita E, Enya M et al. Can endoscopic papillary balloon dilation really preserve sphincter of Oddi function? Gut 2001; 49: 686691

[27] Toouli J. Sphincter of Oddi: Function, dysfunction, and its management. J Gastroenterol Hepatol 2009; 24: (Suppl. 03): S57 - S62

[28] Ishiguro J. Biliary bacteria as an indicator of the risk of recurrence of choledocholithiasis after endoscopic sphincterotomy. Diagn Ther Endosc 1998; 5: 9-17 